\title{
XIST promotes cell proliferation and invasion by regulating miR-140-5p and SOX4 in retinoblastoma
}

\author{
Yuhui Wang ${ }^{1}$, Dahong Sun ${ }^{2}$, Ying Sheng ${ }^{2}$, Hong Guo ${ }^{3}$, Fanchun Meng ${ }^{4}$ and Tingting Song ${ }^{5 *}$
}

\begin{abstract}
Background: Retinoblastoma (RB) is the most common intraocular malignancy in children. Long non-coding RNA $X$-inactive specific transcript (IncRNA XIST) has been reported to be associated with RB, but research on the mechanism of XIST is not well studied.

Methods: Expressions of XIST, microRNA-140-5p (miR-140-5p), and sex-determining region Y-related high-mobility group box 4 (SOX4) were analyzed by qRT-PCR or Western blot. Relationships of XIST, SOX4, and miR-140-5p were evaluated by dual-luciferase reporter assay and Spearman's analysis. Cell Counting Kit-8 (CCK-8) and Transwell assay were performed to assess the function of XIST on RB cell proliferation and invasion.

Results: In RB tissues, XIST and SOX4 expressions were obviously increased, but the miR-140-5p expression was markedly reduced. XIST expression was positively related to SOX4 expression while negatively correlated with miR-140$5 p$ expression, and negative correlation was exhibited between miR-140-5p and SOX4 expression in RB tissues. XIST was confirmed to directly bind to miR-140-5p, and SOX4 was one target of miR-140-5p. XIST knockdown could impede RB cell proliferation and invasion, while miR-140-5p inhibition reversed the effects. In addition, XIST overexpression or miR-140-5p inhibition could abrogate the inhibition of SOX4 silencing on cell proliferation and invasion of RB cells.

Conclusions: XIST was obviously increased in RB tissues and cells, and XIST inhibition repressed the proliferation and invasion of RB cells by miR-140-5p/SOX4 axis, which may provide new understandings of the XIST molecular mechanism in RB.
\end{abstract}

Keywords: Retinoblastoma, XIST, miR-140-5p, SOX4

\section{Background}

Retinoblastoma (RB) is the most common intraocular malignancy in children and rare in adults, with poor prognosis, which seriously affects the vision of children and even endangers life [1]. The etiology of RB is extremely complicated and remains unknown, which may be related to heredity and virus infection [2]. RB accounts for about 3\% of childhood malignancies; in order to improve the survival

\footnotetext{
* Correspondence: pangdmzdobh28626@163.com

${ }^{5}$ No. 2 Department of Oncology, Qingdao Central Hospital, Qingdao Tumor Hospital, 127 Siliu South Road, Shibei District, Qingdao 266042, China Full list of author information is available at the end of the article
}

rate and reduce the rate of eyeball removal, the treatment methods are constantly updated and developed [3]. At present, the main treatment methods are chemotherapy, radiotherapy, surgery, physical therapy, photochemotherapy, gene therapy, and so on [4]. However, the prognosis in life and vision is still not satisfactory. Therefore, it is imperative to explore the pathological mechanism of RB to identify novel biomarkers for RB diagnosis and treatment.

Increasing researches have confirmed that IncRNAs are associated with RB tumorigenesis and progression. For instance, plasmacytoma variant translocation 1 (PVT1) promoted RB progression by its upregulation

C C The Author(s). 2020 Open Access This article is licensed under a Creative Commons Attribution 4.0 International License, which permits use, sharing, adaptation, distribution and reproduction in any medium or format, as long as you give appropriate credit to the original author(s) and the source, provide a link to the Creative Commons licence, and indicate if changes were made. The images or other third party material in this article are included in the article's Creative Commons licence, unless indicated otherwise in a credit line to the material. If material is not included in the article's Creative Commons licence and your intended use is not permitted by statutory regulation or exceeds the permitted use, you will need to obtain permission directly from the copyright holder. To view a copy of this licence, visit http://creativecommons.org/licenses/by/4.0/ The Creative Commons Public Domain Dedication waiver (http://creativecommons.org/publicdomain/zero/1.0/) applies to the data made available in this article, unless otherwise stated in a credit line to the data. 
[5]. Maternally expressed gene 3 (MEG3) was downregulated and suppressed the RB progression [6]. The study of Zhang et al. suggested that H19 was a decreased expression and suppressed RB progression [7], while Li et al. found that $\mathrm{H} 19$ was upregulated in RB and may act as an oncogenic function in RB progression [8]. Xinactive specific transcript (XIST), a widely studied lncRNA, has been reported that it was dysregulated and functioned as an oncogene in RB $[9,10]$. However, our knowledge about detailed mechanisms for XIST in RB is still insufficient.

MiRNAs with about 22 nucleotides length were dysregulated as tumor suppressor or oncogene under certain circumstances in human cancers [11]. Accumulating studies reported that IncRNAs were involved in cancer progression through miRNA-mediated gene regulation by sponging miRNA [12]. miR-140-5p, a miRNA, has been reported as a suppressor in several tumors, like colorectal carcinoma [13], gastric cancer [14], and oral squamous cell carcinoma [15]. Several studies confirmed miR-140-5p also inhibited RB progression as a tumor suppressor [16-18]. However, the specific regulatory mechanisms of it, especially its related lncRNA-miRNAmRNA regulatory networks, are still limited and need to be further explored.

SOX4, one of the sox transcription factors, has been proven as an oncogene and correlated with tumor progression and development $[19,20]$. It has been reported that lots of miRNAs could modulate SOX4 in human cancers [19]. Kooi et al. demonstrated SOX4 as novel RB driver candidates by somatic copy number alteration profiling [21]. At present, research on the function of SOX4 in RB has not been studied still in a deepgoing way by now.

Thereby, XIST, miR-140-5p, and SOX4 expressions and possible mechanisms were explored in $\mathrm{RB}$ in our study. We found that XIST and SOX4 expressions were increased while miR-140-5p was decreased both in RB tissues and cells. In addition, XIST knockdown suppressed proliferation and invasion of $\mathrm{RB}$ cells by miR140-5p/SOX4 axis, indicating XIST might be a potential biomarker for RB treatment and diagnosis.

\section{Materials and methods}

\section{Tissue specimens}

Eight normal retinas and 20 RB tissues were obtained from the Affiliated Hospital of Beihua University. No chemotherapy and local radiotherapy were received before the operation for all patients. This study obtained the Ethical Review of Affiliated Hospital of Beihua University and informed consent of all patients or guardians.

\section{Cell culture}

ARPE-19 (human retinal epithelial cells) and Y79, WeriRb1, SO-Rb50, and HXO-RB44 (human RB cell lines) which were obtained from ATCC were cultured in DMEM (Life Technologies, Carlsbad, USA) media containing $10 \%$ fetal bovine serum (FBS, Gibco, USA). Cells were cultured with $5 \% \mathrm{CO}_{2}$ at a $37^{\circ} \mathrm{C}$ incubator.

\section{Cell transfection}

Si-XIST (small interference RNA of XIST), pc-XIST (XIST-overexpressing plasmid), pc-SOX4 (SOX4-overexpressing plasmid), and miR-140-5p mimic and inhibitor were constructed from GenePharma (Shanghai, China). Lipofectamine 2000 (Invitrogen, USA) was for transient transfection of constructs into cells with the manufacturer's indication.

\section{CCK-8 assay}

Cell proliferation reagent CCK-8 (Roche, Basel, Switzerland) was used to detect cell proliferation in CCK-8 assay. Each group cell was seeded in a 96-well plate (Corning, NY, USA). At 1, 2, 3, and $4 \mathrm{~d}$ after the transfection of each group, $10 \mu \mathrm{L}$ CCK- 8 reagents were added to each well. Subsequently, the 96-well plate was incubated at $37{ }^{\circ} \mathrm{C}$ for $2 \mathrm{~h}$. At last, the values of spectrophotometric absorbance at $450 \mathrm{~nm}$ were detected and recorded.

\section{Cell invasion assays}

Cell invasion was detected using Transwell chamber. Briefly, transfected Y79 were seeded in the upper chambers with a Matrigel-coated and serum-free medium, meanwhile $700 \mu \mathrm{L}$ medium containing $15 \%$ FBS was inserted into the lower chamber. After $48 \mathrm{~h}$ incubation, the outer cells were fixed and then stained with $20 \%$ Giemsa solution. Five random views were selected for cell counting with an inverted microscope (CK2, Olympus).

\section{Dual-luciferase activity assay}

Luciferase reporter vectors including wild-type and mutant-type (XIST-wt, XIST-mut, SOX4-wt, and SOX4mut) were amplified and constructed into the pGL3basic vector (Promega, USA) respectively. RB cells were transfected with miR-140-5p mimics or NC along with the above constructed vectors. After transfection for 48 h, Dual-Luciferase Reporter Assay kit (Promega, USA) was used to measure firefly luciferase activity.

\section{Quantitative real-time PCR}

Total RNA was extracted by TRIzol reagent (Invitrogen, USA), and reverse transcription action was performed by Reverse Transcription Kit (Takara, Japan). qRT-PCR reactions were performed with SYBR Green (Takara) on BioRad CFX96TM System (CA, USA). Primers were listed in Table 1. The expression levels were calculated by the $2^{-\Delta \Delta \mathrm{Ct}}$ method and normalized by U6 and glyceraldehyde phosphate dehydrogenase (GAPDH) expression. 
Table 1 Primer sequences for real-time fluorescence quantification PCR

\begin{tabular}{|c|c|}
\hline Gene name & Primer sequences $\left(5^{\prime}-3^{\prime}\right)$ \\
\hline GAPDH & $\begin{array}{l}\text { F: ACGCTGCATGTGTCCTTAG } \\
\text { R: GAGCCTCTTATAGCTGTTTG }\end{array}$ \\
\hline U6 & $\begin{array}{l}\text { F: CTCGCTTCGGCAGCACA } \\
\text { R: AACGCTTCACGAATTTGCGT }\end{array}$ \\
\hline XIST & $\begin{array}{l}\text { F: AATGACTGACCACTGCTGGG } \\
\text { R: GTGTAGGTGGTTCCCCAAGG }\end{array}$ \\
\hline miR-140-5p & $\begin{array}{l}\text { F: CAGTGGTTITACCCTATGGTAG } \\
\text { R: ACCATAGGGTAAAACCACTGTT }\end{array}$ \\
\hline SOX4 & $\begin{array}{l}\text { F: GGCCTGTTCGCTGTCGGGT } \\
\text { R: GCCTGCATGCAACAGACTGGC }\end{array}$ \\
\hline
\end{tabular}

\section{Western blot}

Total proteins were extracted by RIPA reagent (Beyotime, China) and separated using 10\% SDSPAGE. Subsequently, the bands were transferred onto polyvinylidene difluoride (PVDF) membrane (Sigma-Aldrich, USA) and probed with primary antibodies (SOX4 and GAPDH antibodies, 1:1000 dilution) at $4{ }^{\circ} \mathrm{C}$ overnight. Then, membranes were incubated for another $2 \mathrm{~h}$ with secondary antibodies conjugated to HRP at room temperature. All antibodies were purchased from Abcam (Cambridge, UK). The bands were visualized by enhanced chemiluminescence (ECL) method.

\section{Statistical analysis}

Data are presented as the mean \pm standard deviation (SD). The differences between the groups were compared using Graph Prism 5.0 software (San Diego, CA, USA) with a one-way analysis of variance (ANOVA) or Student $t$ test. $P<0.05$ indicated a statistically significant result in comparison.

\section{Results}

XIST expression was increased in RB tissues and cells

Firstly, we analyzed the XIST expression in RB tissues and found XIST expression was obviously increased in RB tissues versus normal tissues (Fig. 1a). Similarly, an increasing expression of XIST was observed in RB cells (Y79, Weri-Rb1, SO-Rb50, and HXO-RB44) compared to ARPE-19 (human retinal epithelial cells) (Fig. 1b). Among them, the highest XIST expression was found in Y79 cells, so we chose Y79 cells for follow-up experiments (Fig. 1b). These data suggested that dysregulation of XIST may be associated with RB progression.

\section{XIST enhances $\mathrm{Y} 79$ cell proliferation and invasion}

To detect roles of XIST in RB, si-XIST, pc-XIST, or NC scramble was transfected into Y79. Compared with $\mathrm{NC}$, XIST expression was obviously reduced and enhanced in Y79 cells transfected with si-XIST and pc-XIST, respectively (Fig. 2a). Cell proliferation was confirmed to be restrained in Y79 cells transfected with si-XIST and promoted by pc-XIST in CCK-8 assay (Fig. 2b). Moreover, the Transwell assay showed that cell invasion was also inhibited by XIST knockdown and promoted by XIST overexpression (Fig. 2c). Thus, we suspected that XIST may play a potential carcinogenesis in RB.

\section{miR-140-5p directly binds to and negatively correlated with XIST}

To investigate the regulatory mechanism of XIST in RB, prediction of target miRNA was performed by bioinformatics analysis. XIST is predicted to have potential binding sites with miR-140-5p (Fig. 3a). We performed the luciferase activity assays in order to verify the prediction. Luciferase activity was obviously reduced in the cells transfected with XIST-wt and miR-140-5p mimics
A

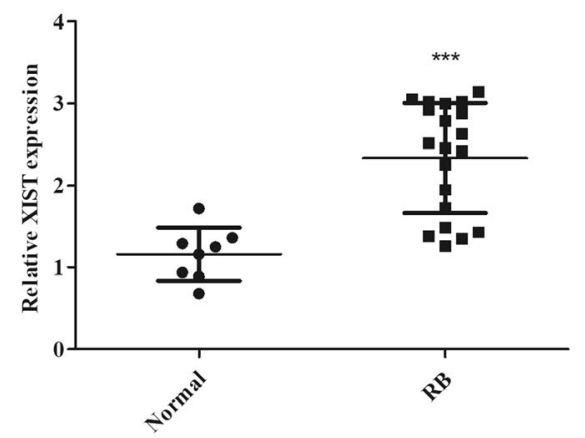

B

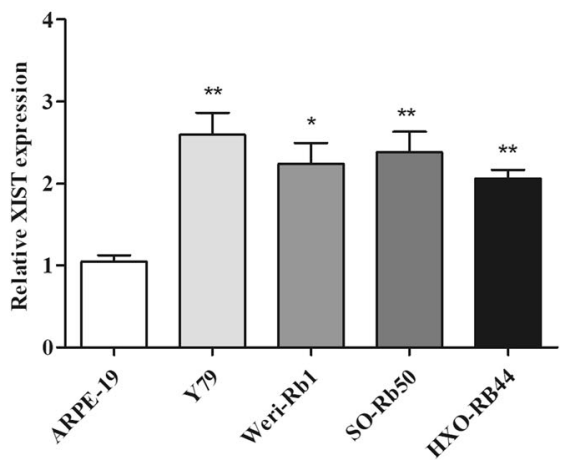

Fig. 1 Expression of XIST was examined in RB tissues and cells. a Expression of XIST was significantly upregulated in RB tissues. $\mathbf{b}$ XIST expression was remarkably upregulated in RB cells compared to human retinal epithelial cells. ${ }^{*} P<0.05$, ${ }^{* *} P<0.01$, and ${ }^{* * *} P<0.001$, compared with normal tissues or the ARPE-19 group 


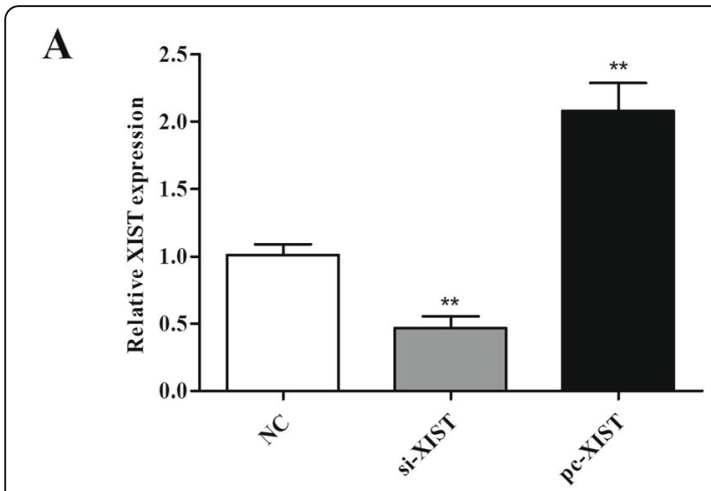

B

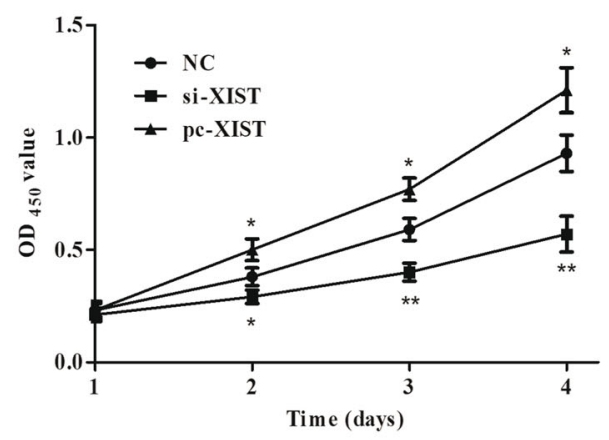

C
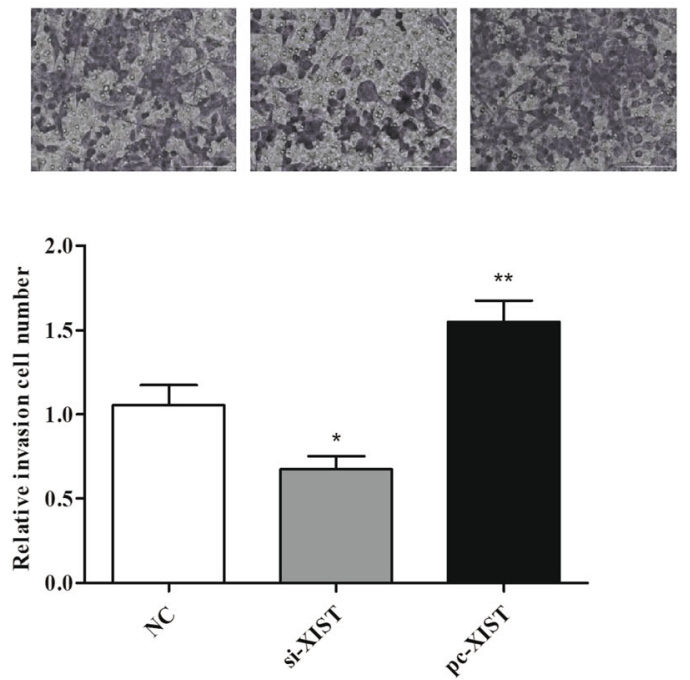

Fig. 2 XIST promoted cell proliferation and invasion in Y79 cells. a XIST expression was decreased or increased in cells after si-XIST or pc-XIST was transfected. $\mathbf{b}$ XIST knockdown or overexpression regulated cell proliferation of Y79 cells. c Cell invasion of Y79 cells was regulated by XIST knockdown or overexpression. ${ }^{*} P<0.05$ and ${ }^{* *} P<0.01$, compared with the NC group

compared with other groups (Fig. 3b). In addition, miR140-5p expression was lower and inversely related to XIST expression in RB tissues (Fig. 3c, d). miR-140-5p expression was reduced after XIST overexpression while reversed by XIST knockdown in Y79 cells (Fig. 3e). To further confirm their relation, miR-140-5p mimics were transfected into Y79 cells along with pc-XIST. The expression of miR-140-5p was increased by its mimics while reduced in the presence of pc-XIST (Fig. 3f). In a rescue experiment, XIST overexpression also attenuated the inhibition of miR-140-5p mimics on cell proliferation and invasion (Fig. 3g, h). Collectively, the results suggested that XIST may promote RB progression by targeting miR-140-5p.

\section{SOX4 is a direct target of miR-140-5p}

We used the TargetScan software to predict miR-140-5p target genes, and SOX4 has binding sites of miR-140-5p (Fig. 4a). Luciferase activity was obviously reduced in Y79 cells with miR-140-5p overexpression and SOX4-wt transfection rather than others (Fig. 4b). Furthermore, SOX4 expressions both in mRNA and protein were downregulated when miR-140-5p was upregulated, while it increased by the inhibition of miR-140-5p in Y79 cells (Fig. 4c). In addition, SOX4 was upregulated in RB tissues versus normal tissues (Fig. $4 \mathrm{~d}$ ), and in RB tissues, it had a negative relationship with miR-140-5p expression (Fig. 4e). Inversely, XIST and SOX4 expression exhibited a positive correlation in RB tissues (Fig. 4f). On the basis of the above results, we speculated that SOX4 may contribute to RB cell proliferation and invasion via XIST/ miR-140-5p.

\section{$\mathrm{XIST} / \mathrm{miR}-140-5 p$ axis promotes cell proliferation and invasion through SOX4}

To test whether XIST can regulate SOX4 via miR-140$5 \mathrm{p}$ in RB, we performed qRT-PCR and Western blot assay. We knocked down SOX4 expression in RB cells using si-RNA. Transfection with si-SOX4 significantly could decrease SOX4 expression, and si-SOX4 and miR140-5p inhibitor or pc-XIST co-transfection increased SOX4 expression (Fig. 5a). Furthermore, the obvious rescue of miR-140-5p inhibitor or pc-XIST on the proliferation and invasion ability induced by si-SOX4 in RB cells were observed in Transwell and CCK-8 assays (Fig. 5b, c). These results indicate that XIST may promote cell proliferation and invasion through the miR-140-5p/SOX4 axis to facilitate RB progression.

\section{Discussion}

$\mathrm{RB}$ is the most frequent intraocular cancer of childhood with highly aggressive and metastatic but a rare disease (about $4 \%$ of all pediatric malignancies) [22]. Interestingly, increasing studies demonstrated that lncRNAs play important roles in promoting or suppressing RB progression, metastasis, diagnosis, and prognosis, such as HOTAIR, NEAT1, XIST, MEG3, and H19 [23]. XIST, a widely studied lncRNA, is dysregulated and serves as a potential biomarker in multiple cancers [24], but research on the mechanism of XIST is not well studied in 
A

miR-140-5p 3' GAUGGUAUCCCAUUUUGGUGAC 5'

XIST-wt 5' ACAUUAaUgugCaUAaCCACUU 3'

XIST-mut 5' ACAUUAAUCUCCUUUUGGUGAU 3 '
B

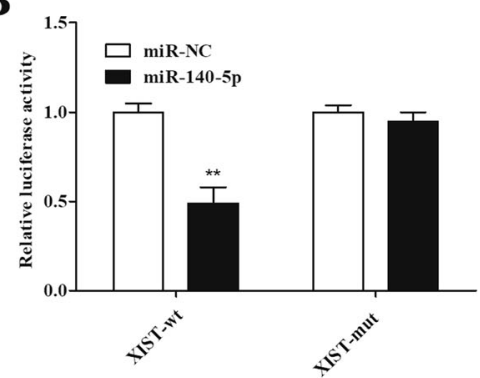

C

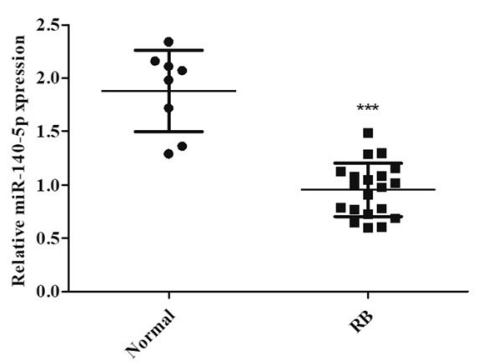

$\mathbf{E}$

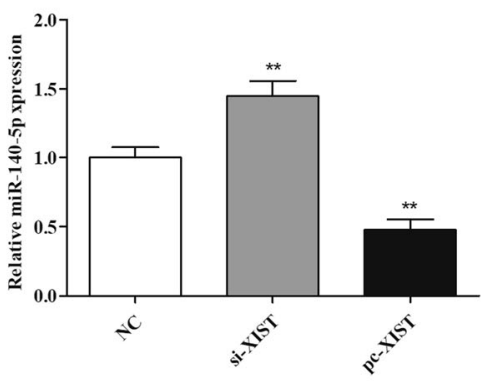

G
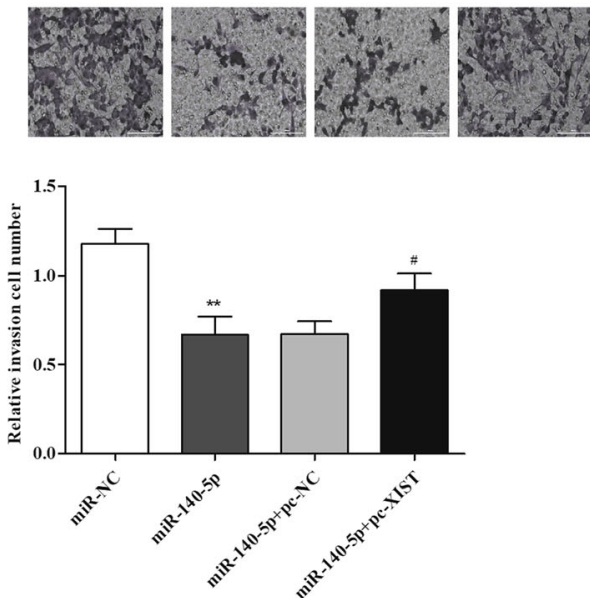

D

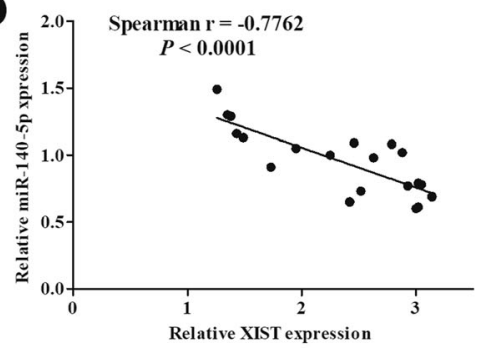

F

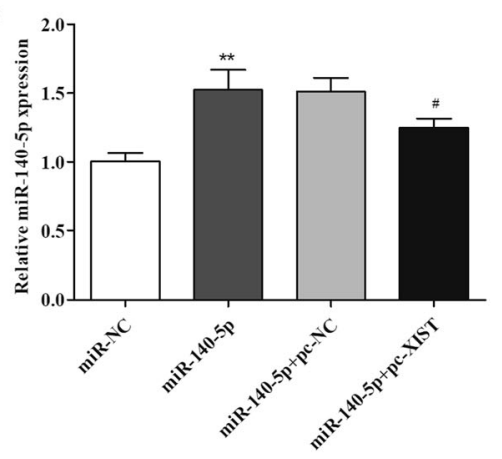

H

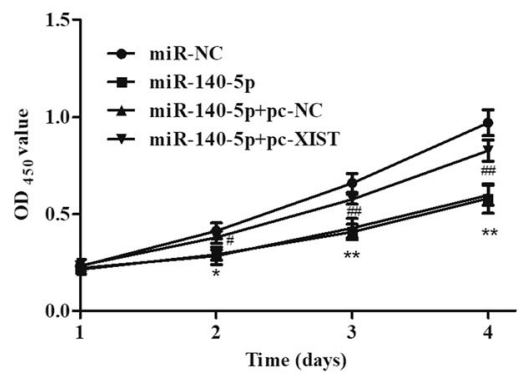

Fig. 3 XIST directly binds to miR-140-5p. a Predicted binding site between miR-140-5p and XIST. b The luciferase reporter assay demonstrated that XIST directly targeted miR-140-5p in Y79 cells. c Expression of miR-140-5p was decreased in RB tissue. $\mathbf{d}$ Correlation analysis between XIST and miR-140-5p in RB tissues. e XIST notably inhibits miR-140-5p expression in Y79 cells. $\mathbf{f}$ Expression of miR-140-5p in Y79 cells transfected with miR-140-5p mimics or miR-140-5p mimics + pc-XIST. g, h Repressive effects of miR-140-5p on cell invasion and proliferation of Y79 cells were attenuated by XIST overexpression. ${ }^{*} P<0.05$, ${ }^{* *} P<0.01,{ }^{\sharp} P<0.05$, and ${ }^{\# \#} P<0.01$, compared with the miR-NC, miR-140-5P+pC-NC, normal, or NC group 


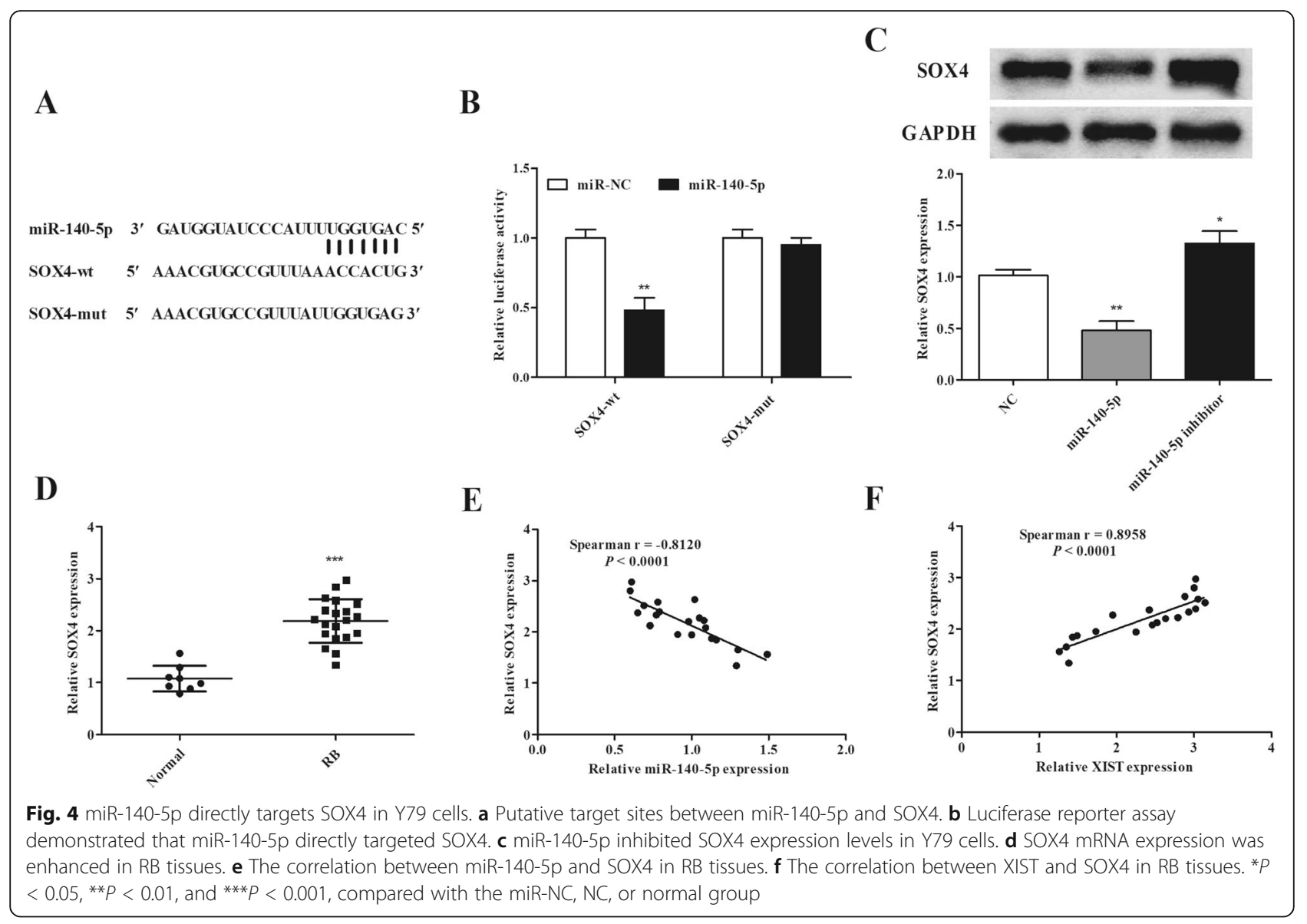

RB. In our study, we explored the roles of XIST and its potential regulatory mechanisms on RB progression. Results revealed that XIST was highly expressed in RB; we proposed it might be served as an oncogene in RB. Functionally, XIST inhibition impeded RB cell proliferation and invasion. Meanwhile, XIST was found to directly bind and negatively regulate miR-140-5p to affect SOX4, thereby promoting RB cell proliferation and invasion capability.

XIST has been reported to be aberrantly expressed and closely related to the progression of various cancers. In pancreatic cancer, XIST contributes to improve cell migration, invasion, and EMT capacities by acting as a miR-429 molecular sponge to regulate ZEB1 expression [25]. Another study has verified that the progression of esophageal cancer was promoted by XIST-regulated miR-494/CDK6 axis to activate the JAK2/STAT3 signal pathway [26]. In thyroid cancer, the XIST/miR-34a axis could promote cell proliferation and tumor growth by regulating MET-PI3K-AKT signaling [27]. Tumor metastasis of colorectal cancer was promoted by the acceleration of the XIST/miR-137/EZH2 axis on cell migration and invasion [28]. Nevertheless, there is no extensive data explaining the function of XIST in RB, except for the finding that XIST promotes the EMT of $\mathrm{RB}$ by modulating miR-101 and conduces to the progression of RB by regulating miR-124/STAT3 axis [9, 10]. All aforementioned findings are in accordance with our results that XIST might serve as an oncogene in RB progression and XIST inhibition could impede RB cell proliferation and invasion.

Additionally, we excavated the specific mechanism of XIST in RB. LncRNAs are reported as miRNAs sponges which can target downstream genes and play vital roles in various cancers progression [29]. A previous study confirmed that in cervical cancer, XIST promoted cell proliferation and suppressed cell apoptosis via miR-140$5 p$ [30]. Moreover, miR-140-5p has been confirmed to inhibit cell proliferation, migration, and invasion while inducing cell apoptosis in RB $[16,17]$. Thus, we proposed that XIST promoted RB cell proliferation and invasion by regulating miR-140-5p. We performed the luciferase reporter assays and confirmed miR-140-5p could directly target XIST. miR-140-5p expression was increased, and a negative association was observed with XIST in RB tissues. Furthermore, in RB cells, miR-140$5 \mathrm{p}$ expression was significantly increased by XIST inhibition while decreased by XIST overexpression. 


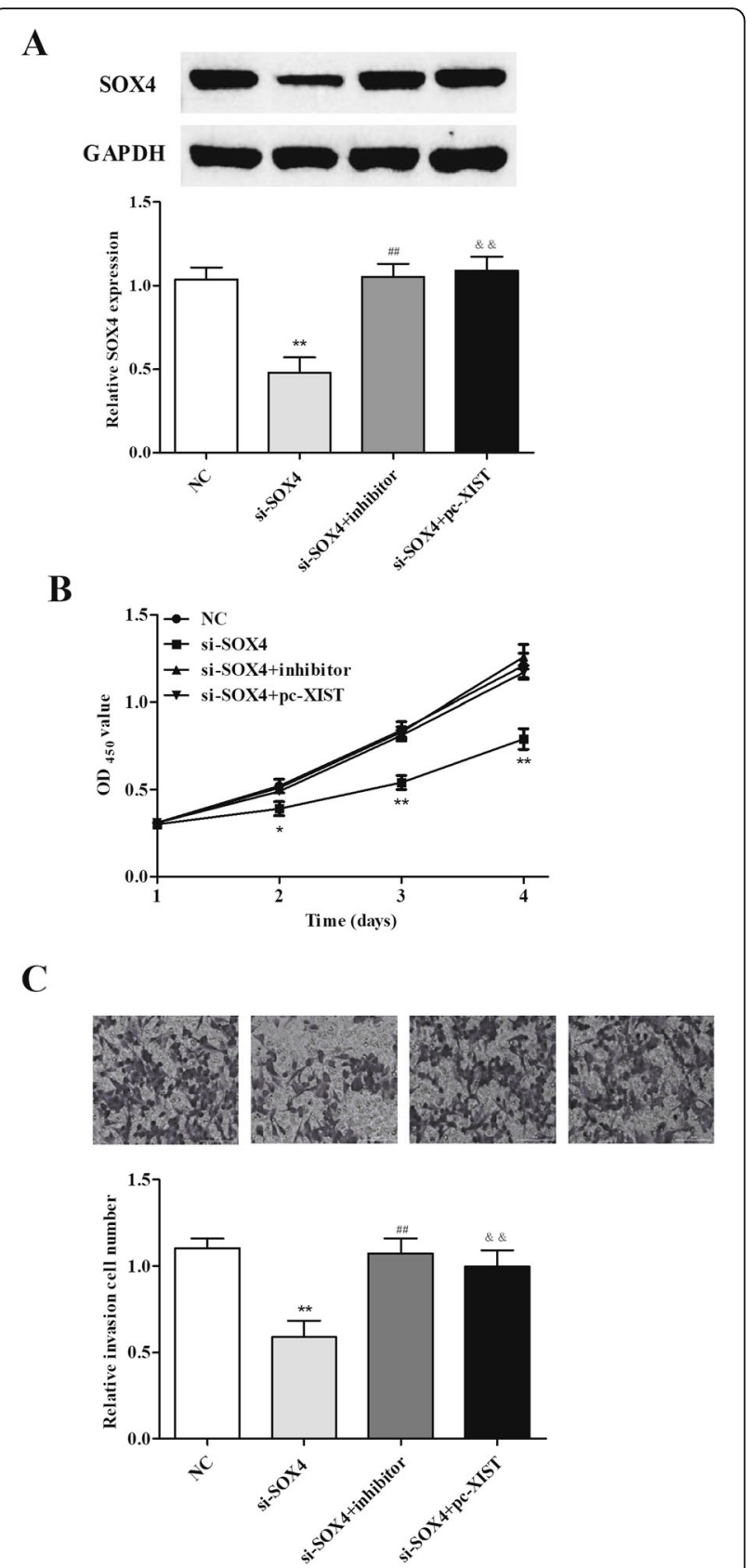

Fig. 5 XIST/miR-140-5p may regulate cell proliferation and invasion through SOX4 in Y79 cells. a SOX4 expression was repressed by siSOX4, and it was attenuated by miR-140-5p inhibitor or XIST overexpression. b, c SOX4 downregulation had a suppressed effect on cell proliferation and invasion of Y79 cells, and it was offset by miR-140-5p inhibitor or XIST overexpression. ${ }^{*} P<0.05$, ${ }^{* *} P<0.01$, ${ }^{\# \# P} P 0.01$, and ${ }^{\& \&} P<0.01$, compared with the NC or si-SOX4 group

Meanwhile, miR-140-5p expression was increased by its mimics while reduced in the presence of XIST overexpression. Finally, XIST overexpression partially abolished the suppression effect of miR-140-5p overexpression on $\mathrm{RB}$ cell proliferation and invasion. In this way, XIST contributed to $\mathrm{RB}$ progression by negatively regulating miR-140-5p.

Subsequently, SOX4 was predicted as one downstream target of miR-140-5p by biological analysis. In malignant melanoma [31] and colorectal carcinoma [13], miR-140$5 \mathrm{p}$ was confirmed to be suppressed cell proliferation and invasion by inhibiting SOX4 expression. Here, we found that SOX4 was upregulated, and its expression has a positive correlation with XIST expression while it was negatively related to miR-140-5p in RB tissues. SOX4 silencing could inhibit RB cell proliferation and invasion, suggesting it may serve as a tumor promoter in RB. More importantly, the inhibition of SOX4 knockdown in cell proliferation and invasion was abolished by miR-140-5p downregulation or XIST upregulation. Overall, our conclusions illustrated that XIST could promote the process of cell proliferation and invasion by modulating miR-140-5p/SOX4 axis, thus accelerating $R B$ progression.

Collectively, we suggested XIST might serve as an oncogene in RB progression by miR-140-5p/SOX4 axis, but still, some limitations are present. First, the function of XIST was not investigated in vivo. Thus, animal experiments (overexpression or silencing) should be carried out. Moreover, we only had a preliminary research on the correlation of XIST and miR-140-5p because of the experimental conditions. Subsequent RNA pulldown or RNA immunoprecipitation experiment should be conducted in cells. Finally, other possible mechanisms of XIST in RB should be explored, such as the effect on cell migration.

\section{Conclusions}

In a word, XIST is closely related to the occurrence and development of RB. XIST inhibition suppressed cell proliferation and invasion of RB cells by the miR-140-5p/ SOX4 axis. Therefore, targeting XIST has great significance in the treatment of RB. However, there are many complicated regulatory pathways involving lncRNAs and miRNAs, and it is necessary to clarify specific signal transduction mechanisms and selectively block key molecules to provide certain guidelines for clinical targeted treatment of RB patients.

\section{Acknowledgements}

Not applicable.

\section{Authors' contributions}

YW and TS designed the experiments. DS, YS, HG, and FM performed the experiments and analyzed the data. YW and TS prepared the manuscript. All authors reviewed, edited, and approved the final manuscript.

Funding

Not applicable. 


\section{Availability of data and materials}

All data generated or analyzed during this study are included in this published article.

\section{Ethics approval and consent to participate}

This study was approved by the institutional review board at the Affiliated Hospital of Beihua University. All the patients and controls were recruited together from the Affiliated Hospital of Beihua University. Written informed consents were obtained from all patients who agreed to serve as tissue donors.

\section{Consent for publication}

Not applicable.

\section{Competing interests}

The authors declare that they have no competing interests.

\section{Author details}

'Department of Clinical Laboratory, Affiliated Hospital of Beihua University, Jilin 132011, China. ${ }^{2}$ Department of Pediatrics, The Third People's Hospital of Qingdao, Qingdao 266041, China. ${ }^{3}$ Health Management Center, The People's Hospital of Zhangqiu Area, Jinan 250200, China. ${ }^{4}$ Delivery Room, The People's Hospital of Zhangqiu Area, Jinan 250200, China. ${ }^{5}$ No. 2 Department of Oncology, Qingdao Central Hospital, Qingdao Tumor Hospital, 127 Siliu South Road, Shibei District, Qingdao 266042, China.

Received: 1 January 2020 Accepted: 19 February 2020

Published online: 03 March 2020

\section{References}

1. Sagoo MS, Shields CL, Shields JA. Retinoblastoma, in Intraocular Inflammation. Springer. 2016:1541-7..

2. Orjuela-Grimm, M., et al. Retinoblastoma: incidence and etiologic factors, in Clinical Ophthalmic Oncology. 2019, Springer. p. 39-56.

3. Rodriguez-Galindo C, Orbach DB, VanderVeen D. Retinoblastoma. Pediatr Clin. 2015;62(1):201-23.

4. Rao R, Honavar SG. Retinoblastoma. Ind J Pediatr. 2017:84(12):937-44.

5. Wu X-Z, et al. Knockdown of IncRNA PVT1 inhibits retinoblastoma progression by sponging miR-488-3p. Biomed Pharmacother. 2019;112: 108627

6. Gao Y, Lu X. Decreased expression of MEG3 contributes to retinoblastoma progression and affects retinoblastoma cell growth by regulating the activity of Wnt/B-catenin pathway. Tumor Biol. 2016;37(2):1461-9.

7. Zhang A, et al. Long non-coding RNA H19 suppresses retinoblastoma progression via counteracting miR-17-92 cluster. J Cell Biochem. 2018;119(4): 3497-509.

8. Li L, et al. Long non-coding RNA H19 regulates viability and metastasis, and is upregulated in retinoblastoma. Oncol Lett. 2018;15(6):8424-32.

9. Cheng $Y$, et al. LncRNA XIST promotes the epithelial to mesenchymal transition of retinoblastoma via sponging miR-101. Eur J Pharmacol. 2019; 843:210-6.

10. Hu C, et al. Knockdown of IncRNA XIST inhibits retinoblastoma progression by modulating the miR-124/STAT3 axis. Biomed Pharmacother. 2018;107: 547-54.

11. Peng $Y$, Croce $C M$. The role of MicroRNAs in human cancer. Signal Transduct Targeted Ther. 2016;1:15004.

12. Kondo Y, Shinjo K, Katsushima K. Long non-coding RNA $s$ as an epigenetic regulator in human cancers. Cancer Sci. 2017;108(10):1927-33.

13. Zhao Z, Liu W, Li J. miR-140-5p inhibits cell proliferation and invasion in colorectal carcinoma by targeting SOX4. Oncol Lett. 2019;17(2):2215-20.

14. Cha $Y$, et al. MicroRNA-140-5p suppresses cell proliferation and invasion in gastric cancer by targeting WNT1 in the WNT/ $\beta$-catenin signaling pathway. Oncol Lett. 2018;16(5):6369-76.

15. Peng, M. and C. Pang, MicroRNA-140-5p inhibits the tumorigenesis of oral squamous cell carcinoma by targeting p21-activated kinase 4. Cell Biology International, 2019

16. Miao X, et al. miR-140-5p suppresses retinoblastoma cell proliferation, migration, and invasion by targeting CEMIP and CADM3. Cell Mol Biol (Noisy-le-Grand). 2018;64(6):42-7.

17. Liao $Y$, et al. MiR-140-5p suppresses retinoblastoma cell growth via inhibiting c-Met/AKT/mTOR pathway. Biosci Rep. 2018;38(6):BSR20180776.
18. Xu C, et al. Long noncoding RNA SNHG16 promotes human retinoblastoma progression via sponging miR-140-5p. Biomed Pharmacother. 2019;117: 109153.

19. Hanieh, $\mathrm{H}_{\text {., }}$ et al. SOX4: epigenetic regulation and role in tumorigenesis. in Seminars in Cancer Biology. 2019. Elsevier.

20. Moreno, C.S. SOX4: The unappreciated oncogene. in Seminars in Cancer Biology. 2019. Elsevier.

21. Kooi IE, et al. A meta-analysis of retinoblastoma copy numbers refines the list of possible driver genes involved in tumor progression. PLoS One. 2016; 11(4):e0153323.

22. Shields CL, Lally SE. Retinoblastoma, in Ocular Oncology. Springer. 2019:91-9.

23. Yang $M$, Wei W. Long non-coding RNAs in retinoblastoma. Pathol Res Pract. 2019;215(8):152435.

24. Zhou Q, et al. Long non coding RNA XIST as a prognostic cancer marker-a meta-analysis. Clinica Chimica Acta. 2018:482:1-7.

25. Shen, J., et al. LnCRNA XIST promotes pancreatic cancer migration, invasion and EMT by sponging miR-429 to modulate ZEB1 expression. The International Journal of Biochemistry \& Cell Biology, 2019

26. Chen Z, et al. Long non-coding RNA XIST promotes the development of esophageal cancer by sponging miR-494 to regulate CDK6 expression. Biomed Pharmacother. 2019;109:2228-36.

27. Liu $\mathrm{H}$, et al. LncRNA XIST/miR-34a axis modulates the cell proliferation and tumor growth of thyroid cancer through MET-PI3K-AKT signaling. J Exp Clin Cancer Res. 2018;37(1):279.

28. Liu X, Cui L, Hua D. Long noncoding RNA XIST regulates miR-137-EZH2 axis to promote tumor metastasis in colorectal cancer. Oncol Res Featuring Preclinical Clin Cancer Ther. 2018;27(1):99-106.

29. Cao M-x, et al. The crosstalk between IncRNA and microRNA in cancer metastasis: orchestrating the epithelial-mesenchymal plasticity. Oncotarget. 2017;8(7):12472

30. Chen $X$, et al. Up-regulated IncRNA XIST contributes to progression of cervical cancer via regulating miR-140-5p and ORC1. Cancer Cell Int. 2019; 19(1):45.

31. Zhao, G., Y. Yin, and B. Zhao, miR-140-5p is negatively correlated with proliferation, invasion, and tumorigenesis in malignant melanoma by targeting SOX4 via the Wnt/nt/o, G., Y. Yin, and B. Zhao, Journal of Cellular Physiology, 2019.

\section{Publisher's Note}

Springer Nature remains neutral with regard to jurisdictional claims in published maps and institutional affiliations.

Ready to submit your research? Choose BMC and benefit from:

- fast, convenient online submission

- thorough peer review by experienced researchers in your field

- rapid publication on acceptance

- support for research data, including large and complex data types

- gold Open Access which fosters wider collaboration and increased citations

- maximum visibility for your research: over $100 \mathrm{M}$ website views per year

At $\mathrm{BMC}$, research is always in progress.

Learn more biomedcentral.com/submissions 